Sharif University of Technology
Scientia Iranica
SCIENTIA

\title{
A new algorithm for the computation of the decimals of the inverse
}

\author{
P. Saha ${ }^{a, *}$ and D. Kumar ${ }^{\text {b }}$ \\ a. Department of Electronics and Communication Engineering, National Institute of Technology Meghalaya, Meghalaya-793003, \\ Shillong, India. \\ b. Department of Computer Science and Engineering, National Institute of Technology Meghalaya, Meghalaya-793003; Shillong, \\ INDIA.
}

Received 3 November 2014; received in revised form 4 December 2015; accepted 27 February 2016

\section{KEYWORDS}

Algorithm;

Arithmetic;

Decimal inverse;

T-Spice;

Propagation delay;

Ancient mathematics.

\begin{abstract}
Ancient mathematical formulae can be directly applied to the optimization of the algebraic computation. A new algorithm used to compute decimals of the inverse based on such ancient mathematics is reported in this paper. Sahayaks (auxiliary fraction) sutra has been used for the hardware implementation of the decimals of the inverse. On account of the ancient formulae, reciprocal approximation of numbers can generate "on the fly" either the first exact $n$ decimal of inverse, $n$ being either arbitrary large or at least $\geq 6$ in almost all cases. The reported algorithm has been implemented, and functionality has been checked in T-Spice. Performance parameters, like propagation delay and dynamic switching power consumptions, are calculated through spice-spectre of $90 \mathrm{~nm}$ CMOS technology. The propagation delay of the resulting 4-digit reciprocal approximation algorithm was only $\sim 1.8 \mathrm{uS}$ and consumed $\sim 24.7 \mathrm{~mW}$ power. The implementation methodology offered substantial reduction of propagation delay and dynamic switching power consumption from its counterpart (NR) based implementation.

(C) 2017 Sharif University of Technology. All rights reserved.
\end{abstract}

\section{Introduction}

Nowadays, decimal computation plays a pivotal role in human-centric areas such as financial and internetbased applications in which exact results are expected. Thereby, hardware implementation of Applying Specific Integrated Circuits (ASICs) has gained popularity during the last decade [1-5]. Generally, hardware implementation of the computer arithmetic circuits is based on binary number systems due to simplicity of operations from decimal number systems [6]. Moreover, lots of decimal numbers cannot be represented exactly in binary format due to finite wordlength effect [6], hence appropriate representation in

*. Corresponding author.

E-mail addresses: sahaprabir1@gmail.com (P. Saha); deepak.enc@gmail.com (D.Kumar) binary format has been impractical. Recently, decimal arithmetic has been presented for general-purposed computer [7] with the help of Binary Coded Decimal (BCD) encoding techniques.

Reciprocal approximation plays a pivotal role for several applications such as digital signal and image processing, computer graphics, scientific computing, etc. [8]. Moreover, division operation can be computed with the help of reciprocal approximation in the following manner: the reciprocal of divisor is computed first, and then it is used as the multiplier in a subsequent multiplication with the dividend [9]. This method is particularly economical when dividend is varying with respect to the same divisor. Nowadays, 'reciprocal approximation' methods are typically based on the Newton-Raphson method [10]. Although, due to its poor performance (high computation time), it is more infrequently used than the other two basic 
arithmetic operations such as addition and multiplication [11].

Substantial amount of algorithms and their hardware implementation has been reported so far [8-12]. All of the algorithms are based on either Taylor series [12] or iterative techniques (Newton-Raphson [9,10] or Gold-Schmidt [11]). These basic algorithms have been amalgamated, and extensive work has been carried out, and then reported by researchers [811]. The mentioned algorithms have long latencies or large area overhead due to its linear convergence rate, thereby a huge number of operations are required for computation. Moreover, the iterative methods start with an initial approximation of the reciprocal of the divisor, usually implemented through a look-up table, thereby large ROM size is required to accommodate the denominator leading to higher delay and power. Thereby, the desired precision level of the reciprocal unit is limited by the ROM size as the size of the lookup table increases with the demand of precision level.

In algorithmic and structural levels, a significant number of algorithms and hardware implementation methodologies have been developed to reduce the propagation delay (latency), which was based on the reduction of the iteration leading to latency reduction, but the principle behind the algorithms are same. In this paper, reciprocal algorithm and its hardware architecture based on ancient mathematics has been addressed. Sahayaks (auxiliary fraction), a Sanskrit term from Vedas, is encountered to realize the reciprocal circuitry. This paper extends the previous paper of the same authors [13] and two others, where the inversion algorithm was introduced for the first time to discuss circuit implementation of a division unit that uses this algorithm. With the help of the ancient methodology, reciprocal algorithm has been realized by algebraic transformation of the digits to smaller ones, and the overall division has been carried out through the transformed digits; thereby, circuit complexity has been reduced substantially due to reduction in propagation delay.

To carry out the transistor level implementation of decimal reciprocal unit, optimized 4221 BCD recording techniques $[14,15]$ have been adopted in this study. The reciprocal unit is fully optimized in terms of calculations; thereby, any configuration of input could be elaborated. Transistor-level implementation of such reciprocal circuitry has been carried out by the combination of $\mathrm{BCD}$ arithmetic with ancient mathematics. Performance parameters, such as propagation delay and dynamic switching power consumption of the reported method, have been calculated by spice/spectre models through $90 \mathrm{~nm}$ CMOS technology and been compared with the other design like Newton-Raphson (NR) [7] based implementation. The calculated results revealed that 4-digit reciprocal units have propagation delay of only $\sim 1.8 \mathrm{uS}$ with $\sim 24.7 \mathrm{~mW}$ dynamic switching power consumption.

\section{Ancient methodology for reciprocal computation}

The gifts of the ancient Indian mathematics in the field of mathematical science are not well recognized. Ancient books offered the mathematical operations which can be carried out mentally to produce fast answers using the sutras. In this paper, sahayaks (auxiliary fraction) for implementing the reciprocal algorithm is presented.

\subsection{Examples}

To fully understand the algorithm, take an example of $\frac{1}{a 9}$, where $a=1,2, \cdots, 9$. In the conversion of such an irregular fraction into recurring decimal, ekadhika (by one more than the previous) process can be used. Assume $a=5$; thus, we want to calculate the value of $\frac{1}{59}$. Hence, ekadhika purva (one more than the previous) is $5+1=6$. The method of the division has been described in Figure 1. The description of the chart implementation procedure is described in Table 1. One more example is given in Appendix A.

\subsection{Algebraic proof of sutra}

Let $x=\frac{q_{0}}{10}+\frac{q_{1}}{100}+\frac{q_{2}}{1000}+\frac{q_{3}}{10000}+\cdots$ be an unknown inverse of number $N$.

If $N=59$, we are going to verify that 6 is the appropriate divisor in the 'avalanche' of Euclidean divisions, $a=b q+r$, where the new dividend is obtained by concatenating the previous rest, $r_{k}$, with the previous quotient, $q_{k}$, (or a function $f\left(q_{k}\right)$ of the

$$
\begin{aligned}
& N=59
\end{aligned}
$$

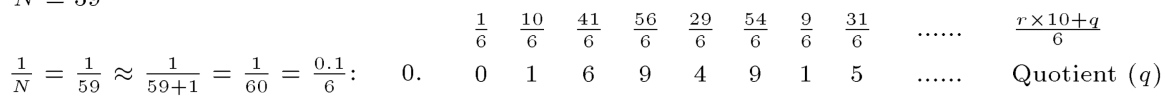

$$
\begin{aligned}
& \begin{array}{llllllllll}
1 & 4 & 5 & 2 & 5 & 0 & 3 & 1 & \ldots \ldots & \text { Remainder }(r)
\end{array} \\
& N=61 \\
& \frac{1}{N}=\frac{1}{61} \approx \frac{1-1}{61-1}=\frac{0}{60}=\frac{0.0}{6}: \quad \begin{array}{llllllllllll} 
& & \frac{0}{6} & \frac{09}{6} & \frac{38}{6} & \frac{23}{6} & \frac{56}{6} & \frac{20}{6} & \frac{26}{6} & \frac{25}{6} & \ldots \ldots & \frac{r \times 10+(9-q)}{6} \\
0 & 0 & 1 & 6 & 3 & 9 & 3 & 4 & 4 & \ldots \ldots & \text { Quotient }(q)
\end{array} \\
& \begin{array}{llllllllll}
0 & 3 & 2 & 5 & 2 & 2 & 2 & 1 & \ldots \ldots & \text { Remainder }(r)
\end{array}
\end{aligned}
$$

Figure 1. Example of reciprocal algorithm: (a) Number is 59 and (b) number is 61 . 
P. Saha and D. Kumar/Scientia Iranica, Transactions D: Computer Science \& ... 24 (2017) 1363-1372

Table 1. Chart implementation procedure of the examples, shown in Figure 1.

\begin{tabular}{|c|c|c|}
\hline \multirow{2}{*}{ Steps } & \multicolumn{2}{|c|}{ Number } \\
\hline & $N=\mathbf{5 9}$ & $N=61$ \\
\hline 1 & $\begin{array}{l}\text { Dividing numerator } 1 \text { by } 60 \text { that is, } \\
1 / 60=0.1 / 6 \text { gives quotient } 0 \text { and } \\
\text { remainder } 1 .\end{array}$ & $\begin{array}{l}\text { Subtract } 1 \text { from numerator and denominator. } \\
\text { Thereby, numerator comes to ' } 0 \text { ' and denominator } \\
\text { comes to } 60 \text {. That is, } 0 / 60 \text { gives } 0.0 / 6 \text {, gives } 0 \\
\text { quotient and remainder } 0 \text {. }\end{array}$ \\
\hline 2 & $\begin{array}{l}\text { Dividing } 10 \text { by } 6 \text { ( } 1 \text { time, remainder } 4 \text { ) } \\
\text { gives the quotient } 1 \text { and remainder } 4 \text {. }\end{array}$ & $\begin{array}{l}\text { Subtract the quotient from } 9 \text { and concatenate } \\
\text { it with the remainder, which gives us } 9 \text {. } \\
\text { Dividing } 9 \text { by } 6 \text { ( } 1 \text { time, remainder } 3 \text { ) } \\
\text { gives quotient } 1 \text { and remainder } 3 \text {. }\end{array}$ \\
\hline 3 & $\begin{array}{l}\text { Dividing } 41 \text { by } 6 \text { ( } 6 \text { times, remainder } 5 \text { ) } \\
\text { gives quotient } 6 \text { and remainder } 5 \text {. }\end{array}$ & $\begin{array}{l}\text { Dividing } 38 \text { by } 6 \text { ( } 6 \text { times, remainder } 2 \text { ) } \\
\text { gives quotient } 6 \text { and remainder } 2 .\end{array}$ \\
\hline 4 & $\begin{array}{l}\text { Dividing } 56 \text { by } 6 \text { ( } 9 \text { times, remainder } 2 \text { ) } \\
\text { gives quotient } 9 \text { and remainder } 2 .\end{array}$ & $\begin{array}{l}\text { Dividing } 23 \text { by } 6 \text { ( } 3 \text { times, remainder } 5 \text { ) } \\
\text { gives quotient } 3 \text { and remainder } 5 \text {. }\end{array}$ \\
\hline 5 & $\begin{array}{l}\text { Dividing } 29 \text { by } 6 \text { ( } 4 \text { times, remainder } 5 \text { ) } \\
\text { gives quotient } 4 \text { and remainder } 5 \text {. }\end{array}$ & $\begin{array}{l}\text { Dividing } 56 \text { by } 6 \text { ( } 9 \text { times, remainder } 2 \text { ) } \\
\text { gives quotient } 9 \text { and remainder } 2 .\end{array}$ \\
\hline 6 & $\begin{array}{l}\text { Dividing } 54 \text { by } 6 \text { ( } 9 \text { times, no remainder) } \\
\text { gives quotient } 9 \text { and remainder } 0 .\end{array}$ & $\begin{array}{l}\text { Dividing } 20 \text { by } 6 \text { ( } 3 \text { times, remainder } 2 \text { ) } \\
\text { gives quotient } 3 \text { and remainder } 2 .\end{array}$ \\
\hline 7 & $\begin{array}{l}\text { Dividing } 09 \text { by } 6 \text { ( } 1 \text { time, remainder } 3 \text { ) } \\
\text { gives quotient } 1 \text { and remainder } 3 \text {. }\end{array}$ & $\begin{array}{l}\text { Dividing } 26 \text { by } 6 \text { ( } 4 \text { times, remainder } 2 \text { ) } \\
\text { gives quotient } 4 \text { and remainder } 2 .\end{array}$ \\
\hline 8 & $\begin{array}{l}\text { Dividing } 31 \text { by } 6 \text { ( } 5 \text { times and remainder } 1 \text { ) } \\
\text { gives quotient } 5 \text { and remainder } 1 \text {, and so on. }\end{array}$ & $\begin{array}{l}\text { Dividing } 25 \text { by } 6 \text { ( } 4 \text { times, remainder } 1 \text { ) } \\
\text { gives quotient } 4 \text { and remainder } 1 \text {, and so on. }\end{array}$ \\
\hline
\end{tabular}

previous quotient, in the general case):

$$
\begin{aligned}
& \left\{\begin{array}{l}
r_{0}=6 q_{0}+r_{0} \\
10 r_{0}+q_{0}=6 q_{1}+r_{1} \\
10 r_{1}+q_{1}=6 q_{2}+r_{2} \\
10 r_{2}+q_{2}=6 q_{3}+r_{3} \\
\text { etc. }
\end{array}\right. \\
& \Rightarrow\left\{\begin{array}{l}
\frac{r_{0}}{10}=6 \frac{q_{0}}{10}+\frac{r_{0}}{10} \\
\frac{r_{0}}{10}+\frac{q_{0}}{100}=6 \frac{q_{1}}{100}+\frac{r_{1}}{100} \\
\frac{r_{1}}{100}+\frac{q_{1}}{1000}=6 \frac{q_{2}}{1000}+\frac{r_{2}}{1000} \\
\frac{r_{2}}{1000}+\frac{q_{2}}{10000}=6 \frac{q_{3}}{10000}+\frac{r_{3}}{10000} \\
\text { etc. }
\end{array}\right.
\end{aligned}
$$

Considering $q_{0}=0$, the right-hand side equations are obtained by dividing the left-hand side equations, especially by $10,100,1000, \cdots$. Then, adding together all these equations, except the first one by canceling and factoring, one obtains:

$$
\frac{r_{0}}{10}+\frac{x}{10}=6 x \Leftrightarrow 59 x=r_{0} .
$$

This equality proves that if we take $r_{0}=1, x$ is the desired inverse of 59 with $q_{k} s$ as its decimals as long as all these $q_{k} s$ are less than 10, which is the case here. The general proof is along the same lines with:

$$
\left\{\begin{array}{l}
r_{0}=d q_{0}+r_{0} \\
10 r_{0}+f\left(q_{0}\right)=d q_{1}+r_{1} \\
10 r_{1}+f\left(q_{1}\right)=d q_{2}+r_{2} \\
10 r_{2}+f\left(q_{2}\right)=d q_{3}+r_{3} \\
10 r_{3}+f\left(q_{3}\right)=d q_{4}+r_{4} \\
\text { etc. }
\end{array}\right.
$$

(here also, $q_{0}=0$ ).

Function $f(.$.$) is to be replaced by one of the$ appropriate rules; for example, if $f_{q k}=2 q_{k}$, one obtains the following by the operation having been done before (division by $10,100,1000, \cdots$ and then adding):

$$
\frac{r_{0}}{10}+2 \frac{x}{10}=d x \Leftrightarrow(10 d+2) x=r_{0}
$$


Taking $r_{0}=2$, one has $(5 d+1) x=1$; thus, we have obtained a recipe for getting all the decimals of the inverse of integers of the form $5 d+1$. Here, $q_{k} \leq 10$. The ancient rule for recipe implementation is given in Appendix B.

\subsection{Implementation of reciprocal algorithm}

Pseudo-code for the implementation of the reciprocal algorithm has been given hereunder. As seen in the pseudo-code, the last digit of the denominator has been calculated through mod-10 operation. If the last digits are 2,4 , and 6 , then it is multiplied by 5 ; if the last digit is 5 , then it is multiplied by 2 . If the last digit is 3 , then it is multiplied by 4 . If the last digit is 0 , then the right-shift operation is directly performed. If the last digit is 1 or " 7,8 , and 9", then the direct implementation of the algorithm is performed. The multiplication process is carried out for digit reduction from denominator. The algorithm will continue until 16 floating point number:

$$
\begin{aligned}
& N \quad / / \text { reciprocal number } \\
& X=10 \quad \text { / Base } 10 \\
& \mathrm{NoD}=\text { Number of digits in Number } N \\
& \text { Num }=1 \quad / / \text { Numerator } \\
& p=0 \\
& \text { Result }=0 / / \text { i.e. reciprocal of } N \\
& \text { Flag }=0 ; F 1=F 2=1 \quad / / \text { flags } \\
& \text { while ( } F 1 \text { AND } F 2 \text { ) } \\
& \mathrm{LSD}=\operatorname{MoD}(N, X) \\
& \text { while ismember(LSD, }[2,3,4,5,6]) \\
& \mathrm{LSD}=\operatorname{MoD}(\mathrm{N}, \mathrm{X}) \\
& \text { Flag }=1 \\
& \text { switch LSD } \\
& \text { case }\{2,4,6\}: m=5 \\
& \text { case 3: } m=4 \\
& \text { case } 5: m=2 \\
& \text { end } \\
& \mathrm{Num}=\mathrm{Num} * m \\
& N=N * m \\
& \text { case }\{7,8,9\}: N=N+X-\mathrm{LSD} \\
& \text { case 0: } \mathrm{Flag}=0 \\
& N=N+X \\
& \text { case } 1: N=N-1 \\
& \text { Num }=\text { Flag } * \text { Num } \\
& N=N / X \\
& p=p+1 \\
& F 1=(\text { Flag }==1) \\
& F 2=\text { Number of digits in } N \geq \operatorname{NoD} \\
& \text { end } \\
& \text { if (NOT } F 1 \text { ) } \\
& \text { for } d=1: 16 \\
& q=\mathrm{Num} / N \quad \text { //integer division }
\end{aligned}
$$

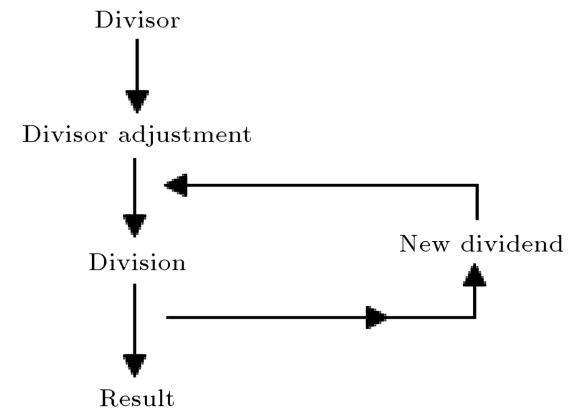

Figure 2. Block level architecture of the reciprocal architecture implementation.

$$
\begin{aligned}
& r=\operatorname{MoD}(\operatorname{Num}, N) \\
& \text { Result }=\text { Result } * X+q \\
& q=\operatorname{MoD}(q, X) \quad \text { //keep single digit } \\
& \text { if } \mathrm{LSD}==1 \\
& \mathrm{Num}=r * X-1-q \\
& \text { else } \\
& \mathrm{Num}=r * X+q *(X-\mathrm{LSD}) \\
& \text { end } \\
& \text { Result }=\text { Result } * X^{-p} \quad / / \text { multiply by } 10^{-p}
\end{aligned}
$$$$
\text { end }
$$$$
p=d
$$$$
\text { end }
$$

\section{Circuit modules}

The proposed reciprocal algorithm technique, shown in Figure 2, is used to implement the hardware architecture. Here, basic block diagram is included. First, the input numbers (i.e., dividend) are taken, and they are forwarded to the divisor adjustment unit. The division adjustment unit consists of comparator, adder, subtractor, and multiplier block. When dividend digits are reduced, then division adjustment unit is promoted for the division circuitry. The block level architecture is shown in Figure 2. Likewise, the reciprocal calculation algorithm is implemented.

\subsection{Divisor adjustment unit}

Divisor adjustment unit is shown in Figure 3, where the input is the divisor and the last digit of the divisor is propagated to the comparator. When the last digit of the divisor is 9 , then ignore the last digit and increment the previous digit by one. When the last digit of the divisor is eight, then ignore the digit and send a

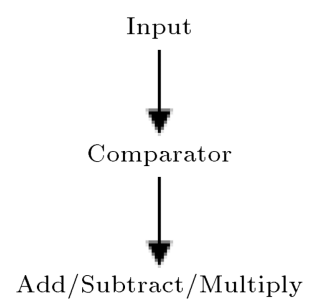

Figure 3. Architecture for divisor adjustment unit. 
signal to a new dividend generation unit for the next iteration. Likewise, when the last digit is 3 , then multiply the numerator and denominator by 4 . When the last digit is equal to 5 , then multiply the numerator and denominator by 2 , then ignore the last digit of the denominator and perform the division.

\subsection{Division}

In this section, divider and hardware implementation algorithms are described to increase the speed of the operation. Where input of the algorithm is initialized as divisor (dvs) and dividend ( $\mathrm{dvd}$ ), and output is given as quotient and remainder. ' $n$ ' is the number of the digits in dividend and ' $i$ ' is the number of iteration. The flow chart for the algorithm is shown in Figure 4. The example of the algorithm is shown in Appendix $\mathrm{C}$ for this algorithm.

The divider implementation technique is shown in Figure 5. The dividend is assumed to have larger length than the divisor length for simplicity. First, the input numbers of the divisor and dividend are taken from the Most Significant Digit (MSD) side. If the MSD of dividend is greater than MSD of divisor, then

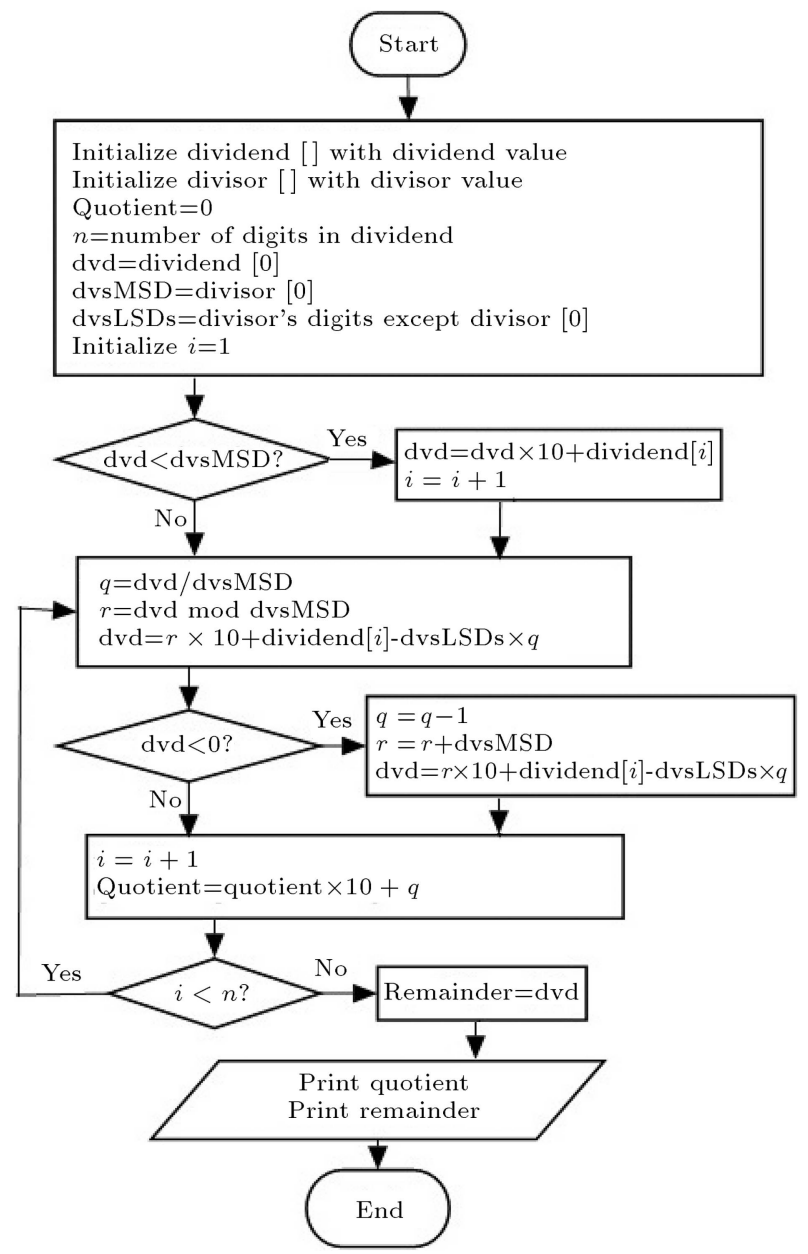

Figure 4. High-speed division implementation of flow chart diagram. divide the dividend MSD by divisor MSD; otherwise, the two most significant digits taken from the dividend side are considered and divided by the divisor. After division, quotient and remainder are generated. The remainder is concatenation of the next MSD of the dividend and subtracted from the multiplication result of the quotient digit and the least significant digit of divisor. If the result is negative, the quotient is reduced by 1 and set the new quotient digit; else it is promoted to the next stage. Likewise, the division algorithm is implemented.

\section{3. $B C D$ computation}

In this paper, we consider the optimized 4221 coding technique for decimal digit representation. As we have mentioned, the use of BCD-8421 to represent decimal digits is expensive because decimal corrections in the partial product reduction binary CSA tree are required to obtain the correct decimal carry and sum. Mathematical representation for the addition technique can be represented as in the following:

$$
\begin{aligned}
& A_{i}+B_{i}+C_{i}=\sum_{i=0}^{3}\left(a_{i, j}+b_{i, j}+c_{i, j}\right) r_{i}=\sum_{j=0}^{3} s_{i, j} r_{j} \\
& \quad+2 \times \sum_{j=0}^{3} h_{i, j} r_{j}=S_{i}+2 H_{i}
\end{aligned}
$$

where $\left(r_{3} \quad r_{2} \quad r_{1} r_{0}\right) \in\{(4221),(5211)\}, s_{i, j}$, and $h_{i, j}$ are the sum and carry bits of full adders, respectively. $H_{i}, S_{i} \in[0,9]$ are the decimal carry and sum digits, respectively. Decimal correction is not required for this 4-bit vector expressions of $H_{i}, S_{i}$ because of coding (4221 and 5211) techniques. Moreover, decimal multiplication by 2 is required for further usage of carry digit, $H_{i}$. The implementation procedure (algorithmic level) for the usage of the carry bit is shown in Figure 6 .

\section{Results and discussions}

The functionality of the proposed algorithm is examined using spice-spectre simulator. Transistorlevel simulation was performed through spice spectre simulator of $90 \mathrm{~nm}$ CMOS technology with ' 1 ' volt node voltage, operated at $250 \mathrm{MHz}$. First, all the circuit modules of full-custom cells are used, and finally the complete architecture that combines all the modules is simulated, so that the decoding performance can be considered and reflected in the results. The simulations have been conducted for all possible bit combinations. The performances, shown in the result section, are the worst-case scenarios when delay and consumed power are maximized for any specific bit combinations. More examples for the calculation of the reciprocal of numbers are given in Appendix C. The reported methodology revealed that the application of 


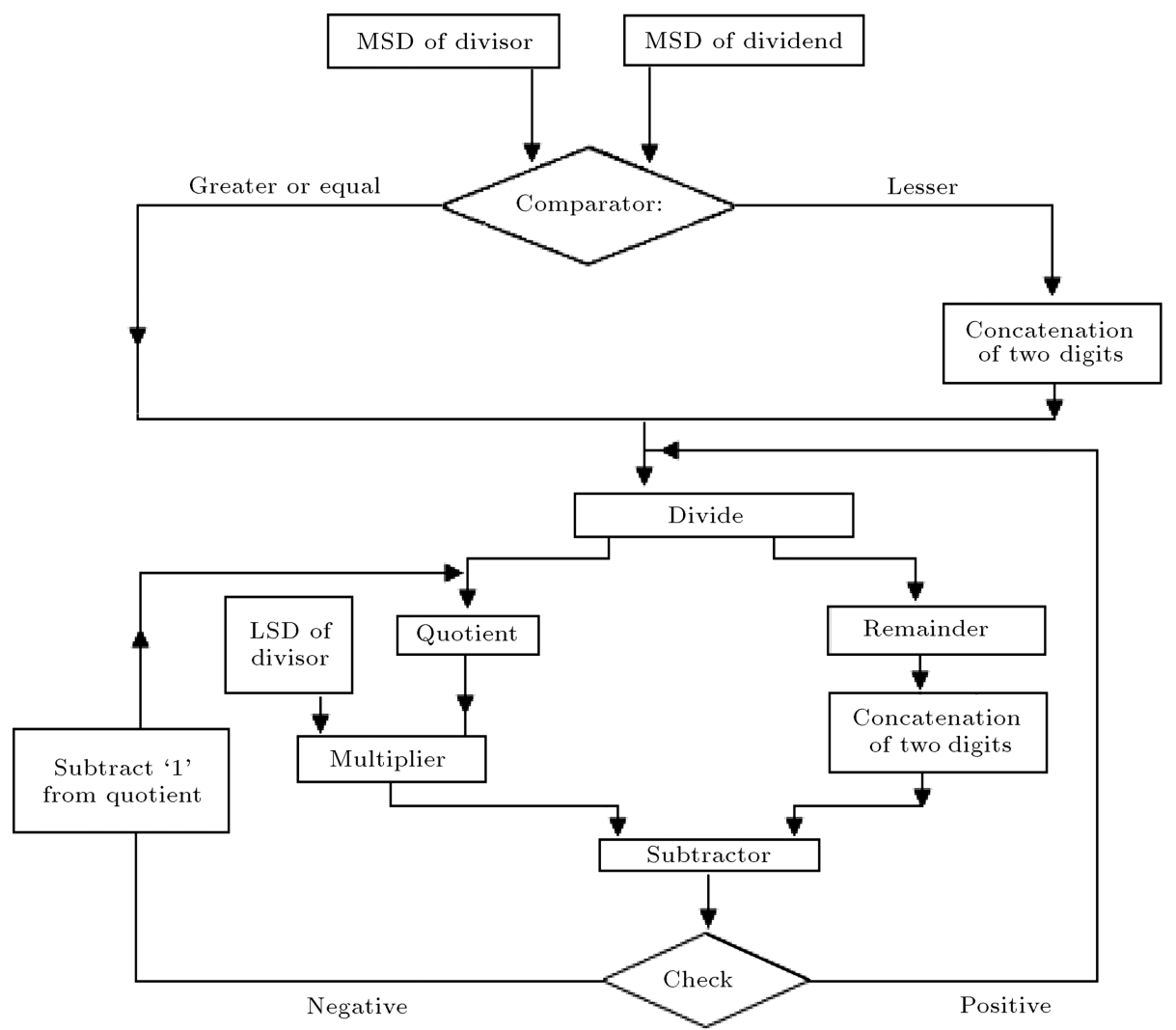

Figure 5. Hardware implementation of divider .

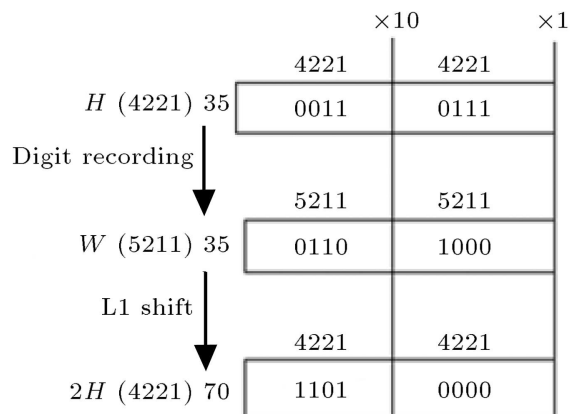

Figure 6. Calculation of carry digit $\left(H_{i}\right)$ based on 4221 coding schemes.

the ancient mathematics for reciprocal implementation reduces the number of iterations. Thereby, hardware usage decreases; as a result, propagation delay and dynamic switching power consumptions decrease.

\subsection{Error analysis}

The computational error can be defined as:

$$
E_{r}=\frac{\text { exact value }- \text { assumed value }}{\text { exact value }} \times 100 \text {. }
$$

The reciprocal of the divisor, $\frac{1}{N}$, is calculated using the Newton-Raphson iterative method. The first iteration uses an initial seed, herein obtained using a piecewise linear approximation based on minimax polynomials. The method converges quadratically, that is, the error of the approximation decreases quadratically with the number of iterations. Calculation of the error in the proposed algorithm is described here:

$$
\frac{X}{Y+k} \cong \frac{X}{Y} \mp \frac{X}{Y}\left(\frac{k}{Y}\right)+\frac{X}{Y}\left(\frac{k}{Y}\right)^{2} \mp \frac{X}{Y}\left(\frac{k}{Y}\right)^{3}+\cdots
$$

Here, the error is:

$$
\begin{aligned}
E_{r} & =\frac{X}{Y+k}-\frac{X}{Y} \\
& \cong \mp \frac{X}{Y}\left\{\left(\frac{k}{Y}\right) \mp\left(\frac{k}{Y}\right)^{2}+\left(\frac{k}{Y}\right)^{3}+\cdots\right\}, \\
& =\mp \frac{X}{Y}\left(\frac{k}{Y}\right)\left\{1 \mp\left(\frac{k}{Y}\right)^{1}+\left(\frac{k}{Y}\right)^{2} \mp\left(\frac{k}{Y}\right)^{3}+\cdots\right\}_{(6)} \\
& \cong \mp \frac{\frac{X}{Y}\left(\frac{k}{Y}\right)}{\left\{1 \pm\left(\frac{k}{Y}\right)\right\}} .
\end{aligned}
$$

Percentage of error is equal to:

$$
\begin{aligned}
E_{r}(\%) & =\frac{E_{r}}{\frac{X}{Y}} \times 100=\frac{\frac{X}{Y+k}-\frac{X}{Y}}{\frac{X}{Y}} \times 100 \\
& \cong \mp \frac{\frac{X}{Y}\left(\frac{k}{Y}\right)}{\frac{X}{Y}\left\{1 \pm\left(\frac{k}{Y}\right)\right\}} \times 100=\mp \frac{\frac{k}{Y}}{1 \pm\left(\frac{k}{Y}\right)} \times 100 .
\end{aligned}
$$




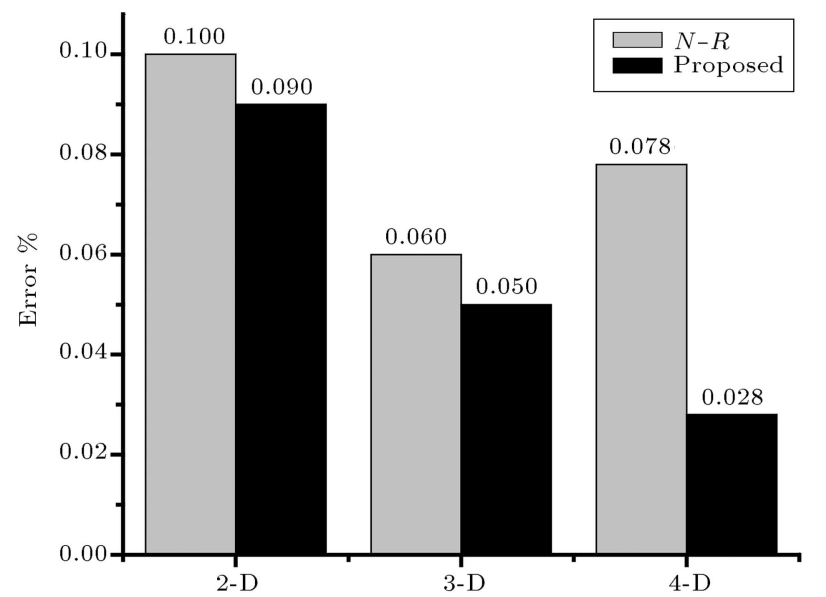

Figure 7. Error analysis chart as a function of input number of digit.

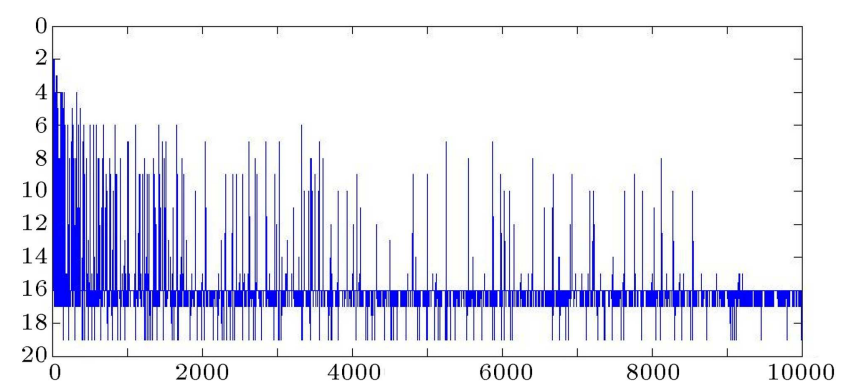

Figure 8. Histogram analysis as a function of input numbers.

Computational error, $E_{r}$, can be minimized if $\left(\frac{X}{Y}\left(\frac{k}{Y}\right)+\right.$ $\left.\frac{X}{Y}\left(\frac{k}{Y}\right)^{2} \mp \frac{X}{Y}\left(\frac{k}{Y}\right)^{3}\right)$ is added to or subtracted from the approximated result. The comparison chart based on the proposed methodology is given in Figure 7 . In Figure 7, comparison of the errors and the N-R methodology are described. MATLAB programming has been implemented using IEEE single precision format, and the value has been calculated for different digits. The error graph shows the approximate and average errors. We have taken the algorithm (N-R) [10] from references and computed it in the same environment for calculation. The number of exact decimals provided by the algorithm is shown in Figure 8 . The histogram analysis, shown in Figure 8, reveals that above (approximately) 400, at least 5 decimals are exact, i.e. the error is under $10^{-6}$.

\subsection{Comparison}

The performance parameters, such as propagation delay and dynamic switching power consumption, are shown in Table 2 as a function of input number of digits. Input data are taken as possible digit combination for experimental purposes. We have kept our main concentration on reducing the performance parameters such as propagation delay, dynamic switching power consumption, thereby energy delay product.

Proper modifications for device, circuit, and ar-
Table 2. Performance parameters' comparison graph as a function of input number of digits: Propagation delay (uS) and dynamic switching power consumption $(\mathrm{mW})$.

\begin{tabular}{cccc}
\hline Algorithm & $\begin{array}{c}\text { No. of } \\
\text { digits }\end{array}$ & $\begin{array}{c}\text { Propagation } \\
\text { delay } \\
\mathbf{( u S )}\end{array}$ & $\begin{array}{c}\text { Power } \\
\mathbf{( m W )}\end{array}$ \\
\hline \multirow{3}{*}{ N-R } & 2-D & 0.52 & 9.63 \\
& 3-D & 1.07 & 14.97 \\
& 4-D & 2.45 & 27.34 \\
\multirow{3}{*}{ Proposed } & 2-D & 0.38 & 7.81 \\
& 3-D & 0.79 & 12.52 \\
& 4-D & 1.83 & 24.76 \\
\hline
\end{tabular}

chitectural levels of design hierarchy have been analyzed properly for reducing propagation delay and average dynamic power consumption. The values of delay and power of different architectures are measured. Pass transistor/Transmission Gates (TG) are used for the design of different modules for faster operation and better logic transformation. The basic difference of pass-transistor logic compared to the CMOS logic style is that the source side of the logic transistor networks is connected to some input signals instead of the power lines. The advantage is that one pass-transistor network (either NMOS or PMOS) is sufficient to perform the logic operation, which results in a smaller number of transistors and smaller input loads, demonstrated in high speed and less power consumption [16]. For each transition, the delay is computed by $50 \%$ of the input voltage swing to $50 \%$ of the output voltage swing.

The propagation delay and the power consumption have been measured with the assumption of the worst-case pattern and from the output where the delay is maximized. Individual circuit module has been simulated, and finally the complete circuit module has been carried out in a similar approach. For comparison purposes, the architectures have been taken from different references $[9,10]$ and implemented using technological environment.

A comparison between different architectures in terms of propagation delay and dynamic switching power consumption is also shown in Table 2. Simulation results for 4-digit reciprocal of a number offered $\sim 25 \%$ speed compared with N-R iteration-based [10] architecture. Moreover, the improvement in terms of switching power is $\sim 9 \%$ in the same environment.

\section{Conclusions}

In this paper, a new algorithm for the computation of the decimals of the inverse based on ancient mathematics is reported. By employing such an ancient methodology, decimal reciprocal has been implemented by the transformation of the digits into a smaller 
one. Moreover, division has been carried out through smaller (transformed) digits. Transformation offered the reduction of circuit-level complexity, owing to the substantial reduction in propagation delay. The functionality of these circuits is checked, and the performance parameters, such as propagation delay and dynamic power consumptions, are calculated through spice spectre of standard 90nm CMOS technology. Simulation results for 4 digits reciprocal of a number offered $\sim 25 \%$ reduction in terms of propagation delay compared with N-R iteration-based [10] architecture, whereas the corresponding improvement in terms of switching power is equal to $\sim 9 \%$ in the same environment.

\section{References}

1. James, R.K., Shahana, T.K., Jacob, K.P. and Sasi, S. "Decimal multiplication using compact BCD multiplier", Proc. IEEE Int. Conf. Electronics Design, Penang, pp. 1-6 (2008).

2. Gorgin, S. and Jaberpur, G. "Comment on high speed parallel decimal multiplication with redundant internal encodings", ", IEEE Transactions on Computers, 64(1), pp. 293-294 (2015).

3. James, R.K., Jacob, K.P. and Sasi, S. "Performance analysis of double digit decimal multiplier on various FPGA logic families", Proc. IEEE Int. Conf. on Programmable Logic, Sao Carlos, pp. 165-170 (2009).

4. Sutter, G., Todorovich, E., Bioul, G., Vazquez, M. and Deschamps, J.P. "FPGA implementations of BCD multipliers", Proc. IEEE Int. Conf. on Reconfigurable Computing and FPGAs (ReConFig '09), Quintana Roo, pp. 36-41 (2009).

5. Zhu, M. and Jiang, Y. "An area-time efficient architecture for $16 \times 16$ decimal multiplications", Proc. of Information Technology: New Generations (ITNG), Las Vegas, pp. 210-216 (2013).

6. Veéstias, M.P. and Neto, H.C. "Parallel decimal multipliers using binary multiplier", Proc. IEEE VI Southern Programmable Logic Conf. (SPL), Ipojuca, pp. $73-78$ (2010).

7. Jaberipur, G. and Kaivani, A. "Binary-coded decimal digit multipliers", IET J. on Computer \& Digital Techniques, 1(4), pp. 377-381 (2004).
8. Schulte, M.J., Stine, J.E. and Wires, K.E. "High-speed reciprocal approximations", Proc. of the Thirty-First Asilomar Conf. on Signals, Systems \& Computers, Pacific Grove, pp. 1183-1187 (1997).

9. Chen, D. and Ko, S.B. "Design and implementation of decimal reciprocal unit", Proc. Canadian Conf. on Electrical and Computer Engineering, Vancouver, pp. 1094-1097 (2007).

10. Fowler, D.L. and Smith, J.E. "An accurate, high speed implementation of division by reciprocal approximation", Proc. 9th Symp. on Computer Arithmetic, Santa Monica, pp. 60-67 (1989).

11. Pineiro, J.A. and Bruguera, J.D. "High-speed doubleprecision computation of reciprocal, division, square root, and inverse square root", IEEE Trans. on Computers, 52(12), pp. 1377-1388 (2002).

12. Farmwald, P.M. "High bandwidth evaluation of elementary functions", Proc. Fifth IEEE Symp. Computer Arithmetic, Ann Arbor, pp. 139-142 (1981).

13. Saha, P., Kumar, D., Bhattacharyya, P. and Dandapat, A. "Reciprocal unit based on Vedic mathematics for signal processing applications", Proc. of the IEEE, Int. Symp. Electronics Design (ISED), Singapore, pp. 41-45 (2013).

14. Bhattacharya, J., Gupta, A. and Singh, A. "A high performance binary to BCD converter for decimal multiplication", Proc. Int. Symp. on VLSI Design Automation and Test (VLSI-DAT), pp. 315-318 (2010).

15. Vazquez, A., Antelo, E. and Montuschi, P. "A new family of high-performance parallel decimal multipliers", Proc. IEEE Symp. on Computer Arithmetic, Montepellier, pp. 195-204 (2007).

16. Zimmermann, R. and Fichtner, W. "Low-power logic styles: CMOS versus pass-transistor logic", IEEE Trans. on Solid State Circuits, 32(7), pp. 1070-1090 (1997).

\section{Appendix A}

One more example is shown in Figure A.1. Chart implementation procedure is described in Table A.1.

$$
\begin{aligned}
& N=789 \\
& \begin{array}{lccccccccccc}
\frac{1}{N}=\frac{1}{789} \approx \frac{1}{789+1}=\frac{1}{790}=\frac{0.1}{79}: & 0 . & 0 & \frac{10}{79} & \frac{100}{79} & \frac{211}{79} & \frac{532}{79} & \frac{586}{79} & \frac{337}{79} & \frac{214}{79} & \ldots \ldots . & \frac{r \times 10+q}{79} \\
\hline & 0 & 1 & 2 & 6 & 7 & 4 & 2 & \ldots \ldots . & \text { Quotient }(q)
\end{array} \\
& \begin{array}{llllllllll}
1 & 10 & 21 & 53 & 58 & 33 & 21 & 56 & \ldots \ldots & \text { Remainder }(r)
\end{array} \\
& N=388 \\
& \begin{array}{lccccccccccc}
\frac{1}{N}=\frac{1}{388} \approx \frac{1}{388+2}=\frac{1}{390}=\frac{0.1}{39}: & 0 . & \frac{1}{39} & \frac{10}{39} & \frac{100}{39} & \frac{224}{39} & \frac{300}{39} & \frac{284}{39} & \frac{124}{39} & \frac{76}{39} & \ldots \ldots . & \frac{r \times 10+q \times 2}{39} \\
& 0 & 2 & 5 & 7 & 7 & 3 & 1 & \ldots \ldots . & \text { Quotient }(q) \\
1 & 10 & 22 & 29 & 27 & 11 & 7 & 37 & \ldots \ldots . & \text { Remainder }(r)
\end{array}
\end{aligned}
$$

Figure A.1. Example of reciprocal algorithm: (a) Number is 789 and (b) number is 388 . 
Table A.1. Chart implementation procedure of the examples, shown in Figure A.1.

\begin{tabular}{|c|c|c|}
\hline \multirow[b]{2}{*}{ Steps } & \multicolumn{2}{|c|}{ Number } \\
\hline & $N=789$ & $N=388$ \\
\hline 1 & $\begin{array}{l}\text { Dividing numerator } 1 \text { by } 790=0.1 / 79 \text { gives } \\
\text { quotient } 0 \text { and remainder } 1 .\end{array}$ & $\begin{array}{l}\text { Dividing numerator } 1 \text { by } 390 . \text { that is } \\
1 / 390=0.1 / 39 \text { gives quotient } 0 \text { and remainder } 1 .\end{array}$ \\
\hline 2 & $\begin{array}{l}\text { Dividing }(r \times 10+q) 10 \text { by } 79(0 \text { times, } \\
\text { remainder } 10) \text { gives the quotient } 0 \text { and } \\
\text { remainder } 10 \text {. }\end{array}$ & $\begin{array}{l}\text { Multiplying quotient with } 2 \text { and remainder with } \\
10(r \times 10+q \times 2) \text { gives } 10 \text {. Dividing } 10 \text { by } 39 \\
\text { gives new quotient } 0 \text { and new remainder } 10 \text {. }\end{array}$ \\
\hline 3 & $\begin{array}{l}\text { Dividing }(r \times 10+q) 100 \text { by } 79 \text { ( } 1 \text { time, } \\
\text { remainder } 21) \text { gives quotient } 1 \text { and remainder } 21 \text {. }\end{array}$ & $\begin{array}{l}\text { Dividing }(r \times 10+q \times 2) 100 \text { by } 39 \text { ( } 2 \text { times, } \\
\text { remainder } 22) \text { gives quotient } 2 \text { and remainder } 22 \text {. }\end{array}$ \\
\hline 4 & $\begin{array}{l}\text { Dividing }(r \times 10+q) 211 \text { by } 79(2 \text { times, } \\
\text { remainder } 53) \text { gives quotient } 2 \text { and remainder } 53 \text {. }\end{array}$ & $\begin{array}{l}\text { Dividing }(r \times 10+q \times 2) 224 \text { by } 39 \text { ( } 5 \text { times, } \\
\text { remainder } 29) \text { gives quotient } 5 \text { and remainder } 29 \text {. }\end{array}$ \\
\hline 5 & $\begin{array}{l}\text { Dividing }(r \times 10+q) 532 \text { by } 79(6 \text { times, } \\
\text { remainder } 58) \text { gives quotient } 6 \text { and remainder } 58 \text {. }\end{array}$ & $\begin{array}{l}\text { Dividing }(r \times 10+q \times 2) 300 \text { by } 39(7 \text { times, } \\
\text { remainder } 27) \text { gives quotient } 7 \text { and remainder } 27 \text {. }\end{array}$ \\
\hline 6 & $\begin{array}{l}\text { Dividing }(r \times 10+q) 586 \text { by } 79(7 \text { times, } \\
\text { remainder } 33) \text { gives quotient } 7 \text { and remainder } 33 \text {. }\end{array}$ & $\begin{array}{l}\text { Dividing }(r \times 10+q \times 2) 284 \text { by } 39(7 \text { times, } \\
\text { remainder } 11) \text { gives quotient } 7 \text { and remainder } 11 \text {. }\end{array}$ \\
\hline 7 & $\begin{array}{l}\text { Dividing }(r \times 10+q) 337 \text { by } 79(4 \text { times, } \\
\text { remainder } 21) \text { gives quotient } 4 \text { and remainder } 21 \text {. }\end{array}$ & $\begin{array}{l}\text { Dividing }(r \times 10+q \times 2) 124 \text { by } 39 \text { ( } 3 \text { times, } \\
\text { remainder } 7) \text { gives quotient } 3 \text { and remainder } 7 \text {. }\end{array}$ \\
\hline 8 & $\begin{array}{l}\text { Dividing }(r \times 10+q) 214 \text { by } 79(2 \text { times and } \\
\text { remainder } 56) \text { gives quotient } 2 \text { and remainder } 56 \text {, } \\
\text { and so on. }\end{array}$ & $\begin{array}{l}\text { Dividing }(r \times 10+q \times 2) 76 \text { by } 39 \text { ( } 1 \text { time, } \\
\text { remainder } 37) \text { gives quotient } 1 \text { and remainder } 37 \text {, } \\
\text { and so on. }\end{array}$ \\
\hline
\end{tabular}

\section{Appendix B}

Ancient rule for recipe implementation:

Let:

$$
\frac{r_{i} \times 10}{D}=\text { quotient } Q_{i}, \text { remainder } r_{i+1} .
$$

then:

$$
r_{i} \times 10=Q_{i} D+r_{i+1} D,
$$

where:

(Dividend $=$ Divisor $\times$ Quotient + Remainder $)$,

$$
\begin{aligned}
& \Rightarrow \frac{r_{i} \times 10}{D}=Q_{i}+\frac{r_{i+1}}{D}, \\
& \Rightarrow \frac{r_{i}}{D}=Q_{i} 10^{-1}+\frac{r_{i+1}}{D} 10^{-1},
\end{aligned}
$$

i.e.:

$$
\frac{r_{i}}{D}=\sum_{i=1}^{\infty} Q_{i} 10^{-i} .
$$

\section{Appendix C}

The example of the divider algorithm is shown in Figure C.1, and chart implementation procedure is shown in Table C.1.
Divisor $=83 \quad$ Dividend $=49873$

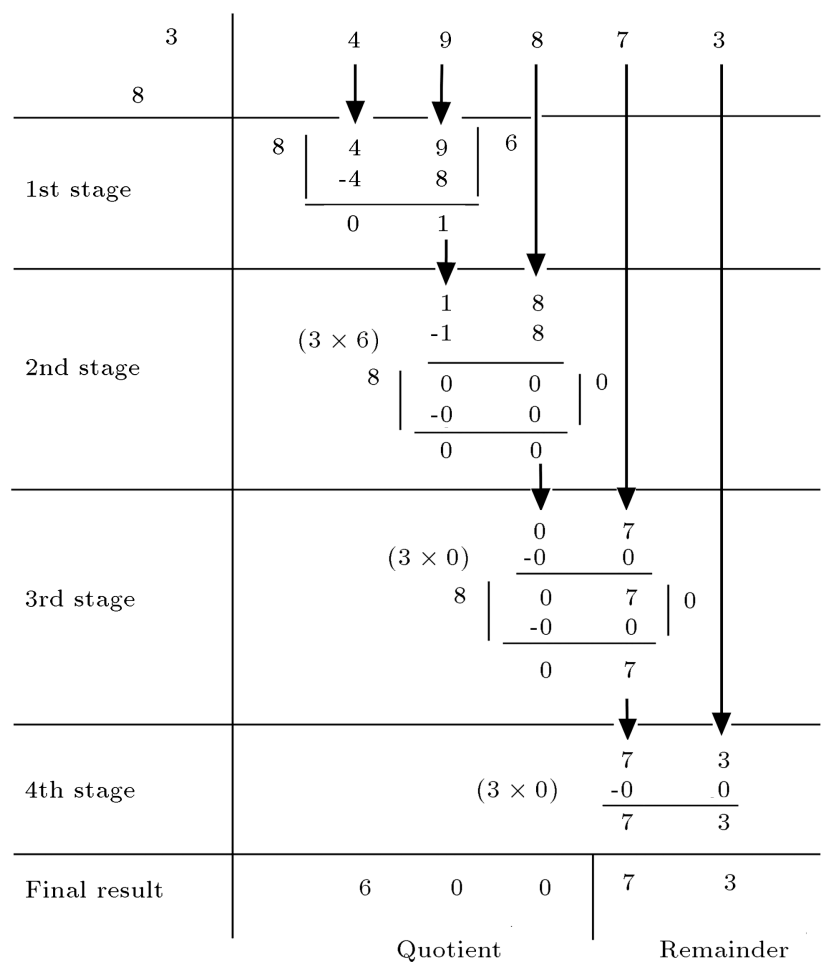

Figure C.1. Division implementation procedure. 
Table C.1. Chart implementation procedure of the examples is considered in Figure C.1.

1) One digit of divisor, i.e., '8' (MSD), has been put a little down compared to the rest of divisor digits (i.e., ' 3 '); this is going to be the actual divisor for subsequent division process. Since 1st digit of dividend (' 4 ') is less than divisor (' 8 '), take two digits of dividend, i.e. ' 49 ', as temporary dividend. After division, we get ' 6 ' as 1 st quotient digit and ' 1 ' as remainder at the completion of 1 st stage.

2) In 2nd stage, temporary dividend (i.e., '00') is generated by concatenating the remainder of 1 st stage (i.e., ' 1 ') with the next unused digit of actual divisor ( i.e. ' 8 ') and subtracting it by the product of the rest of divisor digits, ' 3 ' and quotient digit ' 6 ' of the last stage. After division, we get ' 0 ' as 2 nd quotient digit and ' 00 ' as remainder at the completion of 2 nd stage.

3) In 3rd stage, temporary dividend (i.e., ' 07 ') is generated like in 2 nd stage, and we get ' 0 ' as 3 rd quotient digit and ' 07 ' as remainder at the completion of this stage.

4) In 4th stage, temporary dividend (i.e., '73') is generated like in previous cases. But in this stage, we do not do division and stop the procedure since stopping criteria are met (i.e., the last digit of actual dividend has been used). Thus, we get ' 600 ' as quotient and temporary dividend, '73', as remainder.

\section{Biographies}

Prabir Saha was born in Kolkata, India, in February 1980. He received BTech, MTech, and $\mathrm{PhD}$ degrees in 2003, 2008, and 2014, respectively. Presently, he is working as an Assistant Professor in Electronics and Communication Engineering Department in National Institute of Technology Meghalaya, India. His research interest includes VLSI design, digital signal processing, and digital image processing.
Deepak Kumar was born in Muzaffarpur, India, in January, 1983. He received his BE degree from VTU, Belgaum, in 2007, and ME degree from Bengal Engineering and Science University, Shibpur, in 2009.

Presently, he is pursuing the $\mathrm{PhD}$ degree at National Institute of Technology, Meghalaya, India. His research interest includes computational mathematics, digital signal processing, and digital image processing. 